\title{
Evaluation of Sentinel Lymph Node Biopsy After Primary Chemotherapy as Part of De-Escalation of Breast Cancer Treatment
}

\author{
Mohamed I. AbdelAziz ${ }^{1}$, Sherif Monib ${ }^{2}$ \\ ${ }^{1}$ General Surgery Department, Fayoum University Hospital, Fayoum, Egypt; ${ }^{2}$ St. Albans Hospital \\ Breast Unit, West Hertfordshire Hospitals NHS Trust, Hertfordshire, United Kingdom
}

\begin{abstract}
Background: Sentinel lymph node biopsy (SLNB) after primary chemotherapy for node-positive breast cancer patients has been gaining popularity as part of the de-escalation of treatment.

Aim: The $1^{\text {ry }}$ aim was to assess the surgical outcome of node-positive patients who received primary chemotherapy followed by SLNB. A $2^{\text {ry }}$ aim was to determine the rate of sentinel lymph node identification using the patent blue dye only technique.

Methods: A prospective study that included 86 patients with invasive breast cancer and axillary lymph nodes metastasis as proved by ultrasound scan guided core biopsy. Following the completion of primary chemotherapy, sentinel lymph node biopsy was carried out for all patients at the time of breast surgery using the patent blue dye technique. Patients with negative SLNB underwent no further axillary procedure. Completion of axillary lymph node clearance was performed for positive SLNB patients.

Results: The sentinel lymph node identification rate using the patent blue dye directed technique was $79 \%$. Sixtyseven (78\%) patients underwent wide local excision, and 35\% did not need completion of axillary lymph node clearance due to downstaging following primary chemotherapy.

Conclusions: Sentinel lymph node biopsy following primary chemotherapy for invasive breast cancer appears to be a safe, reliable technique, with an acceptable identification rate, even when using the patent blue dye technique only.

Keywords: Breast cancer, Sentinel lymph node, Neoadjuvant chemotherapy, Patent blue dye Corresponding author: Mohamed AbdelAziz, MD; General Surgery Department, Fayoum University Hospital, Fayoum, Egypt; Email: mohamedibrahim555@hotmail.com

Submitted: 17-April-2021, Finally revised: 29-June-2021, Accepted: 16-July-2021, Published online: 15-January-2022
\end{abstract}

(cc) BY

\section{Introduction}

Breast cancer $(\mathrm{BC})$ is the second most common cancer after lung cancer and the most common cancer in women. It is one of the leading causes of death in females and its incidence, mortality, and survival rates vary considerably among different parts of the world, but in general, on the rise ${ }^{1}$.

It is well accepted that different types of breast cancer respond to different treatment modalities differently depending on tumor biology ${ }^{2}$. Following the establishment of the sentinel lymph node biopsy (SLNB) technique to assess nodal involvement in patients with invasive disease, axillary lymph node clearance (ALNC) became less popular. Axillary lymph node clearance is now only indicated in patients with macrometastasis if they do not have primary chemotherapy, as well as in patients with isolated tumor cells, micrometastasis, or macrometastasis after primary chemotherapy ${ }^{3}$.

Primary chemotherapy has become more popular, especially for triple negative, high-grade, and larger breast cancers as a tool to decrease tumor size, with the aim of breast conservation surgery (BCS) with better outcome ${ }^{4}$. Since primary chemotherapy has been associated with a good axillary nodal response, it is preferable to assess the nodal status after chemotherapy to avoid possible complications and morbidity following ALNC ${ }^{5}$.

The primary aim of this study was to assess the outcome of post-primary chemotherapy sentinel lymph node biopsy, and the secondary aim was to look into our sentinel lymph node identification rate 
using patent blue dye only technique.

\section{Methods}

This prospective study was conducted at the General Surgery Department, Fayoum University Hospital, Fayoum, Egypt from September 2016 to September 2019.

\section{Patients}

The study included adult ( $>18$ years) women with invasive breast cancer and axillary lymph node involvement determined by ultrasound-guided core biopsy who received primary chemotherapy. Exclusion criteria included male gender, inflammatory breast cancer, local recurrence, previous ipsilateral axillary surgery, metastatic disease and non-completion of primary chemotherapy or no response to it.

\section{Management plan}

Initially, all patients underwent a triple evaluation, including clinical breast examination, imaging, and histopathological examination. Imaging included a digital mammogram and a bilateral breast and axillary ultrasound scan, with magnetic resonance imaging performed for a selected group of patients and a staging CT scan performed for all patients. Breast and axilla ultrasound scan was performed before starting primary chemotherapy, after three cycles, and after six cycles to assess the response and help in surgical planning. Some of the lobular cancers or patients with increased breast density on mammogram had breast magnetic resonance imaging as well.

All patients received four cycles of doxorubicin and cyclophosphamide followed by four cycles of paclitaxel. Patients with HER-2 positive disease received four doses of trastuzumab concurrent with paclitaxel, then Herceptin was continued after surgery for one year in total. Pathological complete response (pCR) is defined as the absence of residual invasive cancer on Hematoxylin and Eosin histological evaluation of the breast specimen and sampled axillary lymph nodes following primary systemic therapy.

Sentinel lymph node biopsy was performed for all patients at the time of breast surgery after injecting $2 \mathrm{ml}$ of patent blue into the periareolar region at 10 o'clock. All blue-stained lymph nodes (sentinel lymph nodes) and non-blue palpable (nonsentinel lymph nodes) were harvested. A level one four-node sampling of palpable lymph nodes was performed when we were unable to identify the sentinel lymph node. The harvested nodes were sectioned into 24 sections, and we classified metastatic foci smaller than $0.2 \mathrm{~mm}$ as isolated tumor cells (ITC), foci measuring $0.2-2 \mathrm{~mm}$ as micrometastasis, and foci larger than $2 \mathrm{~mm}$ as macrometastasis.

Patients were classified into two groups: group A, included patients who had negative SLNB, requiring no further axillary procedures, and group B, including patients with positive SLNB (i.e., isolated tumor cells, micrometastasis as well as macrometastasis), requiring completion axillary lymph node clearance. We assessed immediate complications up to 30 days postoperatively and delayed complications up to one year postoperatively and graded them according to the Clavien-Dindo system ${ }^{6}$.

\section{Statistical methods}

The collected data was organized and statistically analyzed using IBM SPSS software, version 22.0. (Armonk, NY: IBM Corp). Quantitative data were presented as mean and standard deviation. Qualitative data were presented as numbers and percentages.

\section{Results}

Eighty-nine patients were included in the study, of which 3 patients were excluded from the analysis (2 patients did not respond to $1^{\text {ry }}$ chemotherapy and 1 patient did not tolerate it). The mean (SD) age of the 86 patients included in the analysis was 58 (12.7) years and they were followed up for a mean of 16.8 months (SD, 4.3). Eighty-four (97.6\%) of these patients presented palpable breast lesions, while 2 (2.4\%) had nonpalpable breast lesions with involved axillary lymph nodes detected on screening mammograms.

Tumor characteristics are illustrated in Table 1. Most of the cancers were found in the upper outer quadrant, and the mean pre-chemotherapy ultrasound scan tumour size was $34 \mathrm{~mm}$ (SD 8). The final histology revealed that most cancers were invasive ductal carcinoma positive for oestrogen and progesterone receptors and 17 (19.8\%) patients had diseases positive for human epidermal growth factor receptor-2 (HER-2). Fifty-four (62.8\%) patients had a Ki67 level $<14 \%$ and 32 (37.2\%) patients had a Ki67 level $<14 \%$.

Table 1: Tumor characteristics 


\begin{tabular}{|c|c|}
\hline Characteristics & $n(\%)$ \\
\hline \multicolumn{2}{|l|}{ Tumor side } \\
\hline Right & $46(53.5)$ \\
\hline Left & $40(46.5)$ \\
\hline \multicolumn{2}{|l|}{ Tumor location } \\
\hline Upper inner quadrant & $17(19.8)$ \\
\hline Upper outer quadrant & $41(47.7)$ \\
\hline Lower outer quadrant & $18(20.9)$ \\
\hline Lower inner quadrant & $10(11.6)$ \\
\hline \multicolumn{2}{|l|}{ Pathology } \\
\hline Invasive ductal carcinoma & $71(82.6)$ \\
\hline Invasive lobular carcinoma & $4(4.6)$ \\
\hline $\begin{array}{l}\text { Invasive disease and ductal } \\
\text { carcinoma in situ }\end{array}$ & $11(12.8)$ \\
\hline \multicolumn{2}{|l|}{ Invasive cancer grade } \\
\hline I & 0 \\
\hline II & $55(64)$ \\
\hline III & $31(36)$ \\
\hline \multicolumn{2}{|l|}{ Ductal carcinoma in situ grade } \\
\hline Low & $3(27.3)$ \\
\hline Intermediate & $3(27.3)$ \\
\hline High & $5(45.4)$ \\
\hline \multicolumn{2}{|l|}{ Molecular type } \\
\hline Luminal A like & $54(62.8)$ \\
\hline Luminal B like (HER2 +ve) & $4(4.7)$ \\
\hline Luminal B like (HER2 -ve) & 0 \\
\hline HER2 overexpression & $13(15.1)$ \\
\hline \multirow[t]{2}{*}{ Triple negative } & $15(17.4)$ \\
\hline & Mean (SD) \\
\hline Tumor size pre-chemotherapy (mm) & $34(8)$ \\
\hline
\end{tabular}

The outline of the management of the included 86 patients is shown in Figure 1.

While we were able to identify the sentinel lymph node in 68 (79.1\%) patients, we carried out level one four-node sampling of palpable lymph nodes in 18 (20.9\%) patients because we were not able to identify the sentinel lymph node. The total number of harvested lymph nodes was 343, of which 196 (57.2\%) were sentinel lymph nodes, 72 (21\%) were part of four-node sampling, and 75 (21.8\%) were non-sentinel lymph nodes. Histopathological evaluation revealed that 67 (19.5\%) lymph nodes were tumor-free, 96 (28\%) had pCR with treatment changes, 38 (11.1\%) had ITC, 45 (13.1\%) had micrometastases, and $97 \quad(28.3 \%)$ had macrometastases.

Group A (node-negative) included 34.9\% (30 patients) who had negative SLNB, requiring no further axillary procedure, while group B (nodepositive) included $65.1 \%$ (56 patients) who had level I, II ALNC. The total number of harvested lymph nodes after clearance was 698, of which 134 (19.2\%) were involved. Sixty-seven (78\%) patients underwent a wide local excision with a mean specimen weight of 76.8 grams (SD: 14.1) and 19 (22\%) underwent mastectomy with a mean specimen weight of 1216.3 grams (SD: 192.4).

All patients treated with wide local excision received adjuvant whole breast radiotherapy and some of those who underwent a mastectomy received chest wall radiotherapy.

Fifty-eight $(67.4 \%)$ patients started adjuvant endocrine treatment (selective estrogen receptor modulators or aromatase inhibitors) for five years with the possibility of extension to ten years in the indicated patients, and 17 (19.8\%) continued trastuzumab for a one-year period in total. The treatment modalities delivered are summarized in Table 2.

\section{Table 2: Treatment modalities}

\begin{tabular}{|c|c|}
\hline Treatment modality & $n(\%)$ \\
\hline \multicolumn{2}{|l|}{ Chemotherapy } \\
\hline Primary chemotherapy only & $69(80.2)$ \\
\hline Primary chemotherapy + trastuzumab & 17 (19.8) \\
\hline \multicolumn{2}{|l|}{ Breast surgery } \\
\hline Breast conserving surgery & $67(78)$ \\
\hline Modified radical mastectomy & $19(22)$ \\
\hline \multicolumn{2}{|l|}{ Axillary surgery } \\
\hline Sentinel lymph node biopsy only & $30(34.9)$ \\
\hline $\begin{array}{l}\text { Sentinel lymph node biopsy followed } \\
\text { by axillary lymph node clearance }\end{array}$ & $56(65.1)$ \\
\hline
\end{tabular}

While Group A patients who had only SLNB with negative lymph nodes did not report any immediate or delayed postoperative complications, Group B patients who underwent ALNC had immediate complications, including seroma $(1 / 56,1.8 \%)$ and minor wound infection (1/56, 1.8\%). Delayed complications included persistent altered sensation at the medial aspect of the arm in 15 (26.8\%) and mild lymphoedema in 4 (7.1\%). None of the patients had limited arm motion. The mean hospital stay was two days. 


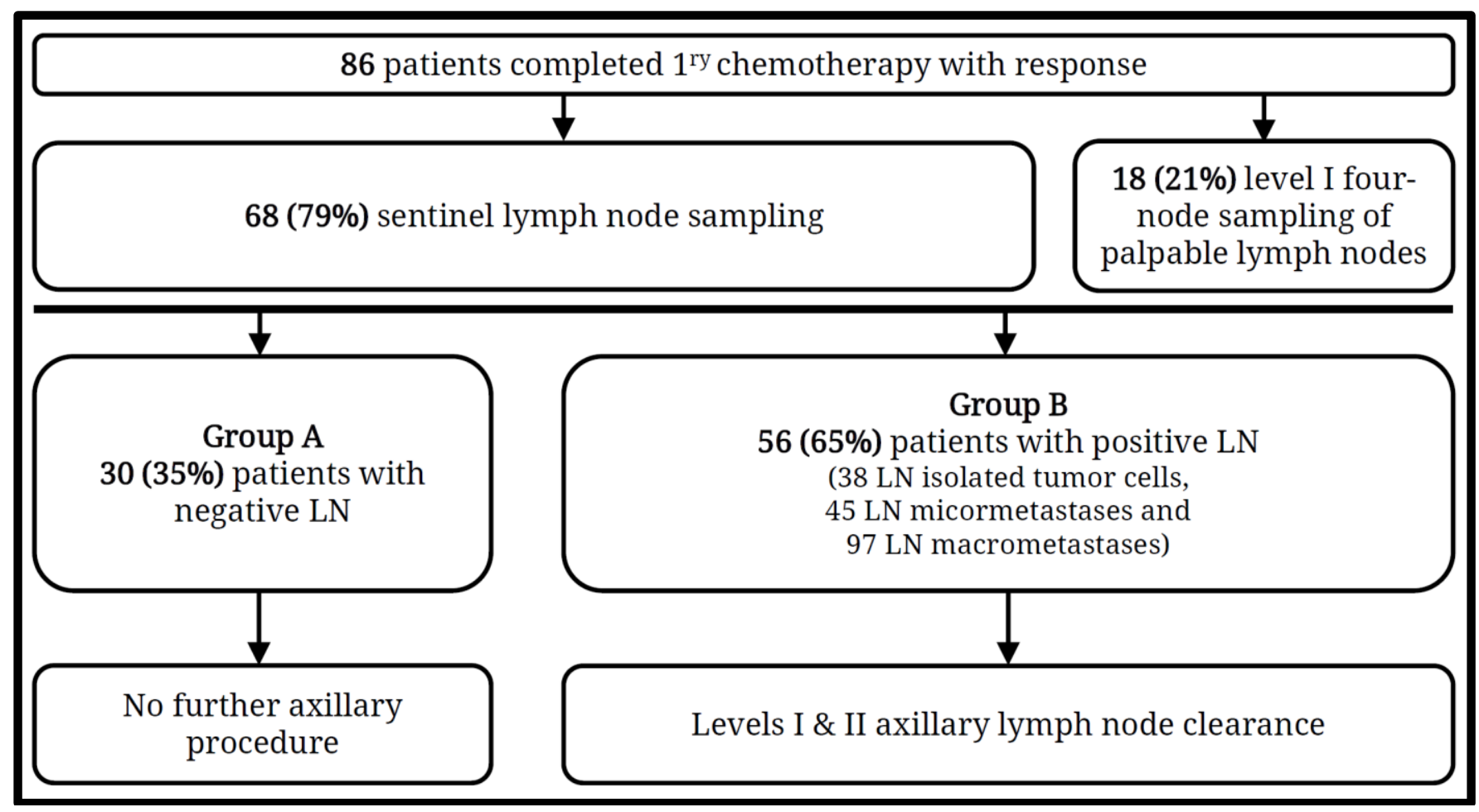

Figure 1: Outline of the management of 86 patients with invasive breast cancer

While 56 (65.1\%) patients had a partial radiological response, 30 (34.9\%) had a complete radiological response on the mid and end of treatment breast ultrasound. The postoperative histological assessment showed a partial response in $60(69.8 \%)$ patients and a complete response in 26 (30.2\%) (26 patients).

\section{Discussion}

Axillary lymph node status does not only direct breast cancer treatment modalities but also plays a vital role in staging and predicting breast cancer prognosis. Hence, axillary lymph node clearance has traditionally been an essential part of the breast cancer management ${ }^{7}$.

Sentinel lymph node biopsy has recently been considered the standard procedure for axillary lymph node histological assessment in clinically node-negative breast cancer and since its introduction, the number of axillary lymph node clearance has significantly decreased ${ }^{8}$.

Recent advances in multimodal treatment have improved breast cancer outcomes and led to a decrease in the magnitude of breast surgery and an increase in the number of breast preservation surgeries after primary chemotherapy, and reduced the number of patients who require axillary clearance. Several studies have confirmed that SLNB after primary chemotherapy may be a feasible and acceptable option to avoid unnecessary axillary dissection ${ }^{9}$.

Unfortunately, traditional imaging and magnetic resonance imaging are not accurate in assessing axillary nodal status after primary chemotherapy ${ }^{10}$. Fowler et al. recommended a combined anatomical and functional/molecular imaging approach as the most accurate method to assess post-primary chemotherapy axillary nodal status ${ }^{11}$.

Post-chemotherapy SLNB is an acceptable and reliable tool to assess the axilla and obtain relative histological findings. It is well known that micrometastasis and isolated tumor cells found after primary SLNB without primary chemotherapy are not considered metastatic disease, as their presence does not affect survival. In contrast, their presence in SLNB after primary chemotherapy is regarded as a residual metastatic disease ${ }^{12}$.

In our cohort, 67 (19.5\%) lymph nodes were tumor-free, 96 (28\%) had pCR with treatment changes, 38 (11.1\%) had ITC, 45 (13.1\%) had micrometastases, and 97 (28.3\%) had macrometastases. There is always a risk of false negative rate with SLNB after chemotherapy which can be attributed to anatomical alterations, interruption or blocking of lymphatic drainage by chemotherapy due to inflammation or fibrosis ${ }^{13}$. Enokido et al. reported a sentinel lymph node 
identification rate of $89.6 \%$ with a false negative rate of $14.2 \%$ after primary chemotherapy ${ }^{14}$. Our sentinel lymph node identification rate was $79.1 \%$.

In the GANEA-2 study, after a 36-month followup, only one patient ( $0.2 \%)$ of 419 patients had an axillary recurrence, with a false negative rate of $11.9 \%{ }^{15}$. The number of sentinel lymph nodes harvested was found to be associated with a decrease in the false negative rate, which was reported to be $7.3 \%$ in the Sentinel Neoadjuvant (SENTINA) study and 9.1\% in the American College of Surgeons Oncology Group (ACOSOG) Z1071 trial when three or more sentinel lymph nodes were retrieved ${ }^{16}$. Mean number of sentinel lymph nodes harvested per patient in our cohort was 2.3 lymph node (196/86)

Rouzier et al. suggested that the axillary nodal status after primary chemotherapy reflects the prognosis more accurately than the initial nodal status ${ }^{17}$. Therefore, axillary lymph node clearance might not be necessary for patients with a complete pathologic response ${ }^{18}$.

Sentinel lymph node biopsy after primary chemotherapy was found to help identify previously positive axillary lymph nodes to a negative status, hence avoiding axillary lymph node clearance leading to reduced arm motion morbidity and lymphedema ${ }^{16}$. In our cohort, 30 (34.9\%) patients did not need axillary clearance due to negative sentinel lymph nodes following primary chemotherapy. After one year, our total arm lymphedema rate was $8.1 \%$ (7/86) and persistent altered sensation at the medial aspect of the arm was observed in 13 (15.1\%) (13/86).

The complete axillary pathological response rate was 35\% in the current study. Choi et al. achieved complete pathologic response of the axillary in $51.9 \%$ of the patients after primary chemotherapy ${ }^{19}$, and Shen J et al. achieved complete pathologic response in the axilla in $30 \%$ of their patients after neoadjuvant chemotherapy. They recommended axillary lymph node clearance for patients with biopsy-determined node metastases regardless of response to neoadjuvant treatment ${ }^{20}$. It is prudent to note that hormone negative status, HER-2 positive disease, and absence of lymphovascular invasion have been associated with a more complete axillary pathologic response ${ }^{10}$.

Despite all available evidence, some authors still insist that omission of axillary lymph node clearance requires identifying at least three sentinel lymph nodes with complete pathological response ${ }^{21}$.

\section{Limitations}

Only 86 patients were included in our analysis, with a short follow-up time. Therefore, a study with longer follow-up and a larger sample size is needed to be able to assess the recurrence rate and survival.

Unfortunately, we did not have the recourses for radioactive directed SLNB. This is the reason why we used the patent blue directed technique.

\section{Conclusions}

Sentinel lymph node biopsy following primary chemotherapy for invasive breast cancer disease appears to be a safe reliable technique, with a good identification rate even when using patent blue dye technique only. Further studies with a larger sample size and longer follow-up comparing patent blue dye technique to dual technique will provide more information to achieve the optimum outcome.

\section{Acknowledgment \\ None.}

\section{Authors' contribution}

Conception or design: MA \& SM; Acquisition, analysis, or interpretation of data: MA; Drafting the manuscript: MA \& SM; Approval of the manuscript version to be published: All authors; Agreement to be accountable for all aspects of the work: All authors.

\section{Conflict of interest}

The authors declare that they have no conflict of interest to disclose.

\section{Data a vailability}

Deidentified individual participant data used to produce the results of this study are available from the corresponding author (MA) upon request.

\section{Ethical considerations}

The study was approved by the Research Ethics Committee of the Faculty of Medicine, Fayoum University. Written informed consent had been obtained from all patients.

\section{Funding}

The authors did not receive funding for this study.

\section{Study registration}

None.

\section{References}

1- Momenimovahed Z, Salehiniya H. Epidemiological characteristics of and risk factors for breast cancer in the world. Breast Cancer (Dove Med Press). 2019; 11:151-164.

2- Waks AG, Winer EP. Breast cancer treatment. JAMA. 2019; 321(3):316.

3- Jatoi I, Benson JR, Toi M. De-escalation of axillary surgery in early breast cancer. Lancet Oncol. 2016; 17(10): e430-e441.

4- Shirzadi A, Mahmoodzadeh H, Qorbani M. Assessment of sentinel lymph node biopsy after 
neoadjuvant chemotherapy for breast cancer in two subgroups: Initially node negative and node positive converted to node negative-A systemic review and meta-analysis. J Res Med Sci. 2019; 24: 18.

5- Boughey JC, Suman VJ, Mittendorf EA, et al. Sentinel lymph node surgery after neoadjuvant chemotherapy in patients with node-positive breast cancer: the ACOSOG Z1071 (Alliance) clinical trial. JAMA. 2013; 310(14): 1455-1461.

6- Dindo D, Demartines N, Clavien PA. Classification of surgical complications: a new proposal with evaluation in a cohort of 6336 patients and results of a survey. Anna Surg. 2004; 240(2): 205-213.

7- Patten DK, Zacharioudakis KE, Chauhan H, et al. Sentinel lymph node biopsy after neo-adjuvant chemotherapy in patients with breast cancer: Are the current false-negative rates acceptable? Breast. 2015; 24(4): 318-320.

8- Kim BK, Park BW, Hur MH, et al. Omission of axillary lymph node dissection in patients who underwent total mastectomy with 1 or 2 metastatic lymph nodes. Ann Surg Treat Res. 2020; 98(6): 283-290.

9- Mocellin S, Goldin E, Marchet A, Nitti D. Sentinel node biopsy performance after neoadjuvant chemotherapy in locally advanced breast cancer: A systematic review and meta-analysis. Int J Cancer. 2016; 138(2): 472-480.

10- Elamin G, Sapre D, Tehniyat W, Jahan A, Dakka M. Pathological complete response in the axillary lymph nodes post neoadjuvant chemotherapy in breast cancer, is it predictable? Ann Breast Surg. 2019; 3: 20.

11- Fowler AM, Mankoff DA, Joe BN. Imaging neoadjuvant therapy response in breast cancer. Radiology. 2017; 285(2): 358-375.

12- Geng C, Chen X, Pan X, Li J. The feasibility and accuracy of sentinel lymph node biopsy in initially clinically node-negative breast cancer after neoadjuvant chemotherapy: A systematic review and meta-analysis. PloS one. 2016; 11(9): e0162605.

13- Park S, Lee JE, Paik HJ, et al. Feasibility and prognostic effect of sentinel lymph node biopsy after neoadjuvant chemotherapy in cytology-proven, node-positive breast cancer. Clin Breast Cancer. 2017; 17(1): e19-e29.

14- Enokido K, Watanabe C, Nakamura S, et al. Sentinel lymph node biopsy after neoadjuvant chemotherapy in patients with an initial diagnosis of cytologyproven lymph node-positive breast cancer. Clin Breast Cancer. 2016; 16(4): 299-304.

15- Classe JM, Loaec C, Gimbergues P, et al. Sentinel lymph node biopsy without axillary lymphadenectomy after neoadjuvant chemotherapy is accurate and safe for selected patients: the GANEA 2 study. Breast Cancer Res Treat. 2019; 173(2): 343352.

16- Choi HJ, Kim I, Alsharif E, et al. Use of sentinel lymph node biopsy after neoadjuvant chemotherapy in patients with axillary node-positive breast cancer in diagnosis. J Breast Cancer. 2018; 21(4): 433-441.

17- Rouzier R, Extra JM, Klijanienko J, et al. Incidence and prognostic significance of complete axillary downstaging after primary chemotherapy in breast cancer patients with $\mathrm{T} 1$ to T3 tumours and cytologically proven axillary metastatic lymph nodes. J Clin Oncol. 2002; 20(5): 1304-1310.

18- Kang YJ, Han W, Park S, et al. Outcome following sentinel lymph node biopsy-guided decisions in breast cancer patients with conversion from positive to negative axillary lymph nodes after neoadjuvant chemotherapy. Breast Cancer Res Treat. 2017; 166(2): 473-480.

19- Choi HJ, Ryu JM, Kim I, et al. Prediction of axillary pathologic response with breast pathologic complete response after neoadjuvant chemotherapy. Breast Cancer Res Treat. 2019; 176(3): 591-596.

20- Shen J, Gilcrease MZ, Babiera GV, et al. Feasibility and accuracy of sentinel lymph node biopsy after preoperative chemotherapy in breast cancer patients with documented axillary metastases. Cancer. 2007; 109(7): 1255-1263.

21- Mamtani A, Barrio AV, King TA, et al. How often does neoadjuvant chemotherapy avoid axillary dissection in patients with histologically confirmed nodal metastases? Results of a prospective study. Ann Surg Oncol. 2016; 23(11): 3467-3474. 